\title{
GLAUCOMA IMAGE CLASSIFICATION USING DISCRETE ORTHOGONAL STOCKWELL TRANSFORM
}

\author{
Gokul Kannan K, \\ Department of Electronics and Communication Engineering, \\ Anna University, \\ Chennai, India \\ gokulkannanme@gmail.com \\ Ganeshbabu T R, \\ Department of Electronics and Communication Engineering, \\ Muthayammal Engineering College, \\ Namakkal, India \\ ganeshbabutr@gmail.com
}

\begin{abstract}
Glaucoma is an eye condition which is caused by the improved blood pressure in the optic nerve. It causes a functional failure of the visual field and irreversible. A Computer Aided Diagnosis (CAD) can help the doctors to find glaucoma at the earliest. In this paper, a CAD system for glaucoma diagnosis using Discrete Orthogonal Stockwell Transform (DOST) is presented. DOST distribute its coefficients based on sample spacing paradigm where low frequencies have a lower sampling rate, and high frequencies have higher sampling rate. All DOST coefficients are considered for the diagnosis of glaucoma using Random Forest (RF) classifier. Results show that the glaucoma diagnosis system has 96\% sensitivity, 92\% specificity, and 94\% accuracy using 100 fundus images of normal and glaucoma cases.
\end{abstract}

Keywords: Fundus Image, Glaucoma, Discrete Orthogonal Stockwell Transform, Random Forest Classifier.

\section{INTRODUCTION}

The diagnosis of glaucoma at the earliest is vital for effective treatment. Haralick texture features based automated diagnosis of glaucoma is presented in [1]. Texture features by the Haralick approach and K-Nearest Neighbors (KNN) classifier are analyzed for glaucoma diagnosis. Glaucoma diagnosis by texture and higher order spectra features is discussed in [2]. To execute supervised classification, sequential minimal optimization, Bayesian classifiers, RF, and Support Vector Machine (SVM) are used.

Glaucoma detection based on Discrete Wavelet Transform (DWT) is described in [3] by the use of optical coherence tomography images. With the help of t-test class separability criteria, significant wavelet coefficients are selected after DWT decomposition, and for automated diagnosis, these features are fed into the SVM classifier. Survey about automatic classification and detection of glaucoma using fundus images is described in [4]. Variety of risks and eye diseases are detected using this procedure. Size measurement, neuro retinal rim and optic cup shape are involved in glaucoma identification.

Wavelet energy features based glaucoma classification is explained in [5]. From each sub-bands of wavelet transformed fundus images, texture features are extracted based on the energy distribution and a probabilistic neural network is used to classify them. Daubechies, symlets, and bi-orthogonal wavelet filters are 
used to extract features. The obtained energies from all sub-band coefficients are used to differentiate between glaucomatous and normal images.

Diagnosis of glaucoma using digital fundus images is presented in [6]. At first, the person's eye colour is plotted by the use of database images which consists of normal as well as abnormal. The observation will show whether the person is affected or not with the help of colour pixels in the affected area. Wavelet and moment feature based automatic classification of glaucomatous images is discussed in [7]. The image decomposition is obtained by using three wavelet filters; bi-orthogonal, Symlets (sym3) and Daubechies (db3). In the feature computation step, higher order moments are extracted. Before classification, zscore normalization is applied to normalize the extracted higher order moments of the normal and abnormal fundus image. Three classifiers are employed for classification viz. KNN, error back-propagation training algorithm, and SVM.

Texture analysis for glaucoma classification is explained in [8]. An independent elementary features based binary robust texture features are extracted. Finally, SVM classifier is used for the classification. Wavelet transform based glaucomatous image classification is described in [9]. DWT is used for feature extraction. The significant texture features are found in the distribution energy over the wavelet sub-bands. Wavelet features are obtained from different wavelet filters; db3, sym3, and reverse bi-orthogonal.

Texture features based classification of glaucoma using the neural network is presented in [10]. They are extracted and restricted around the optic cup. By using these features, the classification of glaucomatous images is achieved using a neural network classifier.

Glaucomatous image classification based on Independent Component Analysis (ICA) of retina images is described in [11]. Image analysis is based on ICA approach, and then classification is achieved using KNN classifier. Cross validation algorithm based glaucoma detection is explained in [12]. Classification, clustering, fuzzy decision tree, association are used.

In this paper, DOST based glaucomatous image classification system is presented. The organization of this paper is as follows: Section 2 gives the methods and materials used in this study. Section 3 gives the results of the glaucoma classification system, and section 4 concludes the glaucoma classification system by DOST and RF classifier.

\section{METHODS AND MATERIALS}

The glaucoma image classification system is shown in Figure 1 which shows the flow of the extraction of DOST features and classification step. Features are extracted after preprocessing the fundus image which includes the Region Of Interest (ROI) extraction. Fundus image in the dataset is subjected to DOST technique for feature extraction. The extracted DOST features stored in the database. Then, in the classification stage, RF classifier is used.

\section{A. Preprocessing}

It is an essential stage in the glaucoma image classification system. At first, the conversion of RGB to Gray is done to separate the colour components of input fundus image. Based on the maximum intensity value in the green channel component, a centre point is chosen and then $360 \times 360$ pixels sized ROI is extracted around the centre pixel in the entire colour components. Figure 2 shows the original input image and their corresponding RGB colour components. 


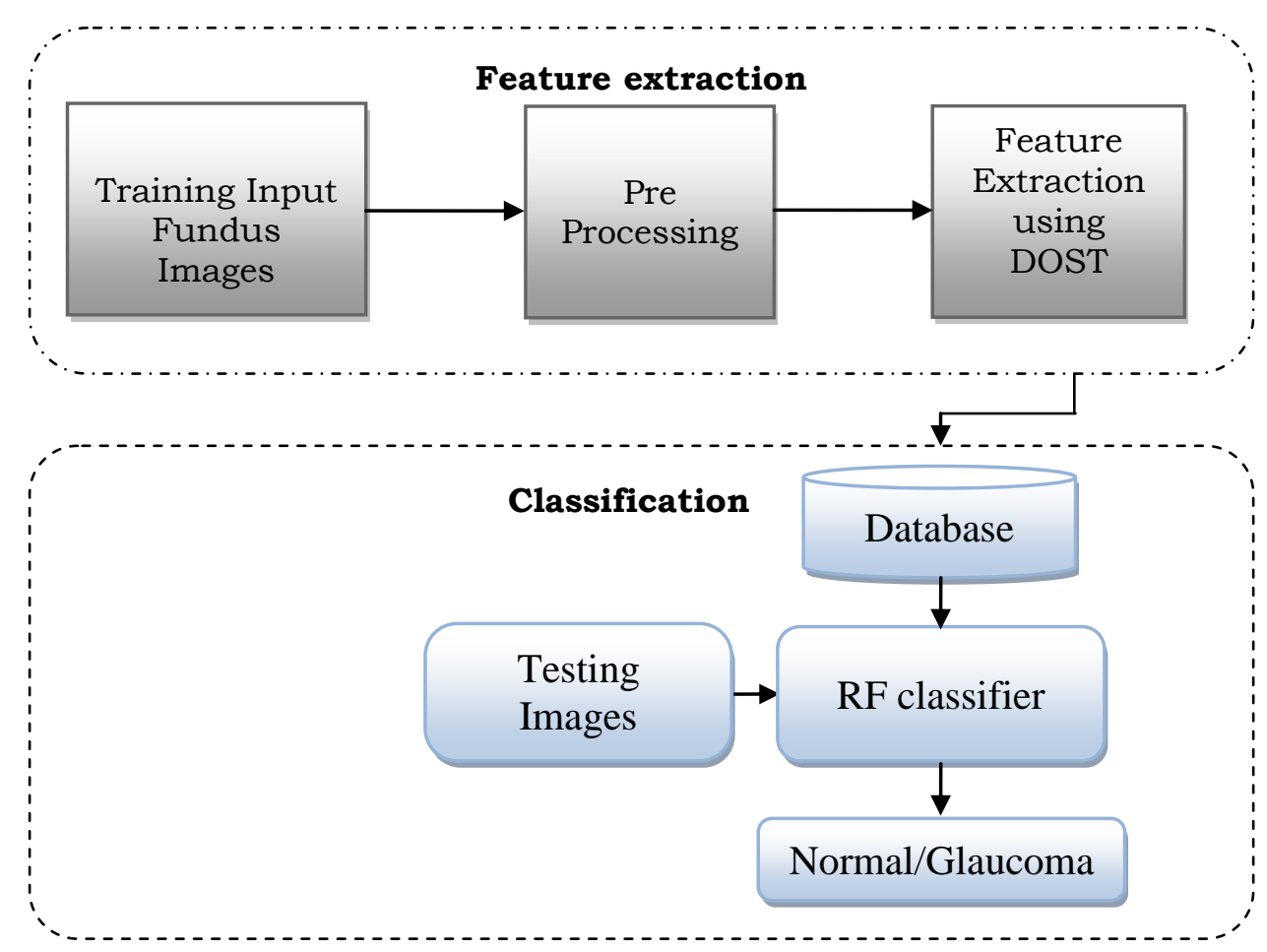

Fig. 1 Fundus image classification system using DOST and RF classifier

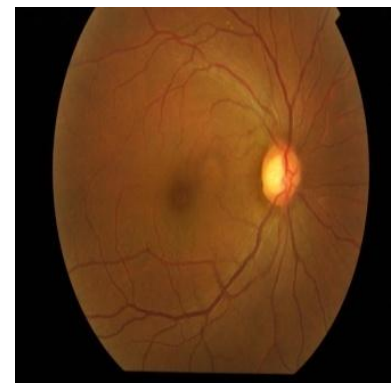

(a)

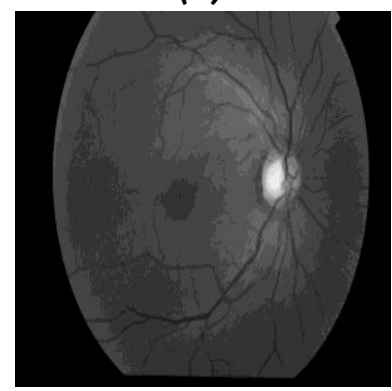

(c)

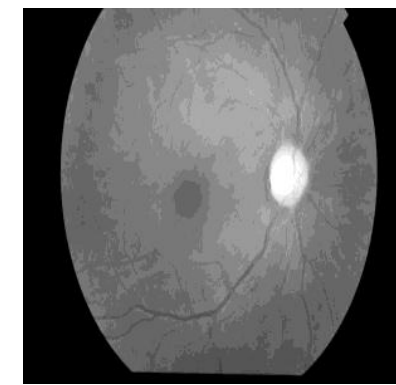

(b)

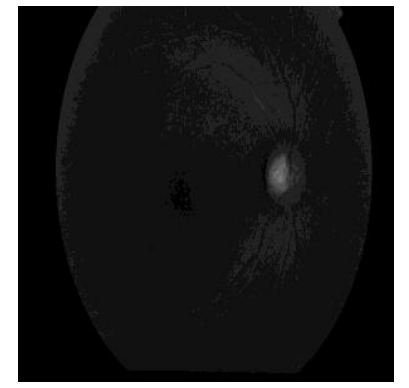

(d)

Fig. 2 (a) Fundus of a normal case (b) Colour component- Red (c) Colour component- Green (d) Colour component- Blue 


\section{B. DOST Features}

The DOST technique [13] is used to extract the feature which is a multi scale technique. The basis for DOST technique is S-transform. It has a timefrequency representation which is very close to the time-frequency representation of continuous wavelet transform. The major limitations of S-transform due to its redundant nature are high computational time and complexity of space. Hence, orthonormal set of basic functions is used by DOST to remove these limitations. While comparing with S-transform, the set of orthonormal basic function provides less computational and storage complexity than S-transform.

It is a pared-down version of S-transform which is fully redundant. Since the low-frequency components have longer periods, they are sub sampled by DOST. Likewise, high-frequency components are subsampled by DOST with higher sampling rates. DOST approaches distribute its coefficients consequently by taking the advantage of sample spacing paradigm.

\section{Classification}

To check the validation of the extracted DOST features for the classification of fundus images, RF classifier which is a very popular machine learning method is used. RF classifier is mainly used for many learning tasks, classification as well as regression analysis and also it is appropriate for classification of the dataset having a large number of samples. A group of treestructured classifiers is called as RF classifier.

While training, it constructs a multitude of decision trees with the class variable at its node. Before fitting each node, the training features are projected into a randomly generated sub-space. Finally, the decision is made by the idea of randomized node optimization.

\section{RESULTS AND DISCUSSION}

An internal database is used to analyze the glaucoma image classification system discussed in section II. It consists of 200 colour fundus image. Among them, 100 normal images and 100 glaucoma images are available. The original size of the fundus image is $1504 \times 1000$ pixels. Figure 3 shows a sample normal and abnormal (glaucoma) image in the database. After preprocessing, the size of the extracted ROI is $360 \times 360$ pixels.

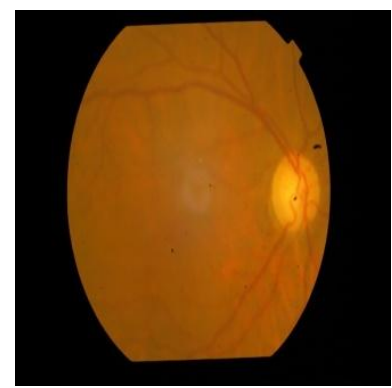

(a)

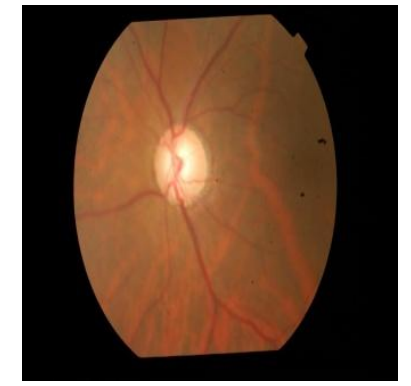

(b)

Fig. 3 (a) Normal fundus image (b) Abnormal fundus image 
To access the performance, the confusion matrix is computed based on the results of the system. Table 1 illustrates the confusion matrix for a two-class problem; class 1 (glaucoma case) and class 2 (normal case). It is constructed using the actual class with the test outcome.

TABLE 1 Confusion matrix of glaucoma image classification system

\begin{tabular}{|c|c|c|}
\hline \multirow{2}{*}{$\begin{array}{c}\text { Test } \\
\text { outcome }\end{array}$} & \multicolumn{2}{|c|}{ Actual Class } \\
\cline { 2 - 4 } $\begin{array}{c}\text { Positive } \\
\text { (glaucoma) }\end{array}$ & $\begin{array}{c}\mathbf{9 6} \text { - True Positive - glaucoma } \\
\text { image is correctly classified as } \\
\text { glaucoma }\end{array}$ & $\begin{array}{c}\mathbf{8} \text { - False Positive - normal } \\
\text { image is incorrectly classified } \\
\text { as glaucoma }\end{array}$ \\
\hline $\begin{array}{c}\text { Negative } \\
\text { (normal) }\end{array}$ & $\begin{array}{c}\mathbf{4}-\text { False Negative - glaucoma } \\
\text { image is incorrectly classified as } \\
\text { normal }\end{array}$ & $\begin{array}{c}\mathbf{9 2} \text { - True Negative - normal } \\
\text { image is correctly classified as } \\
\text { normal }\end{array}$ \\
\hline
\end{tabular}

Using the computed confusion matrix in Table 1, sensitivity and specificity are computed, and the obtained measures are shown in Figure 4.

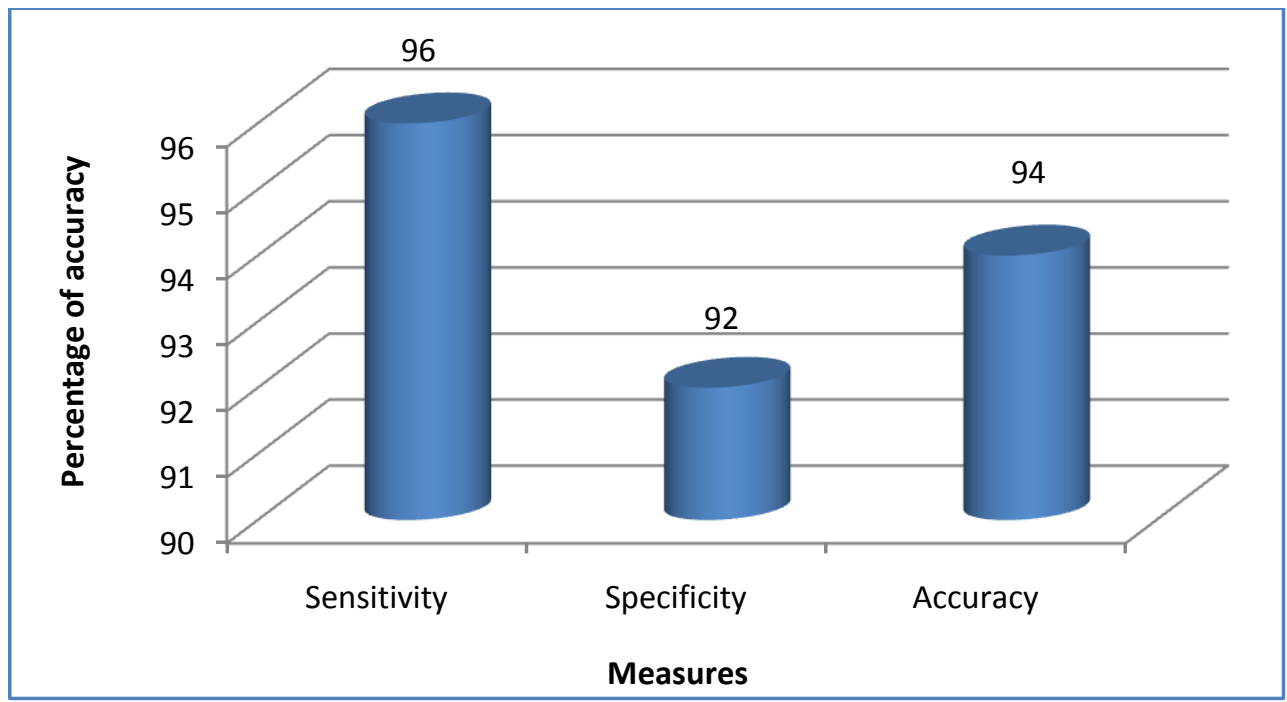

Fig. 4 Performance of glaucoma image classification system

\section{CONCLUSION}

This paper demonstrates an automated glaucoma classification by the use of DOST and RF classifier. Fundus images are used for the glaucoma diagnosis. Features are computed automatically using DOST technique and results show that a high level of accuracy is obtained using these features. A total of 200 fundus images (100 normal and 100 glaucoma) are used. The glaucoma image classification system is evaluated regarding accuracy, specificity, and sensitivity. The sensitivity of the system is $96 \%$ which shows that only four images of glaucoma are misclassified as normal. 


\section{REFERENCES}

[1]. S. Simonthomas, N. Thulasi, and P. Asharaf, "Automated diagnosis of glaucoma using Haralick texture features", IEEE International Conference on Information Communication and Embedded Systems, 2014, pp. 1-6.

[2]. U.R. Acharya, S. Dua, X. Du, and C.K. Chua, "Automated diagnosis of glaucoma using texture and higher order spectra features", IEEE transactions on Information Technology in Biomedicine, Vol.15, No.3, 2011, pp.449-455.

[3]. A. Rajan, and G.P. Ramesh, "Automated Early Detection of Glaucoma in Wavelet Domain Using Optical Coherence Tomography Images", Biosciences Biotechnology Research Asia, Vol.12, No.3, 2015, pp.28212828.

[4]. S.A. Hussain, and A.N. Holambe, "Automated Detection and Classification of Glaucoma from Eye Fundus Images: A Survey", International Journal of Computer Science and Information Technologies, Vol.6, No.2, 2015, pp. 1217-1224.

[5]. N. Annu, and J. Justin, "Automated classification of glaucoma images by wavelet energy features", International Journal of Engineering and Technology, Vol.5, No.2, 2015, pp.1716-1721.

[6]. A.N.S. Byahatti, K. Sridevi, and R. Hegadi, "Computer Based Diagnosis of Glaucoma using Digital Fundus Images", Proceedings of the World Congress on Engineering, Vol. 3, 2014, pp. 3-5.

[7]. G.O. Gajbhiye, and A.N. Kamthane, "Automatic classification of glaucomatous images using wavelet and moment feature", IEEE India Conference, 2015, pp. 1-5.

[8]. S. Mohammad, and D.T. Morris, "Texture analysis for glaucoma classification. IEEE International Conference on BioSignal Analysis, Processing and Systems, 2015, pp. 98-103.

[9]. A. Rajan, G.P. Ramesh, and J. Yuvaraj, "Glaucomatous image classification using wavelet transform", IEEE International Conference on Advanced Communication Control and Computing Technologies, 2014, pp. 1398-1402.

[10]. D. Yadav, M.P. Sarathi, and M.K. Dutta, "Classification of glaucoma based on texture features using neural networks", IEEE Seventh International Conference on Contemporary Computing, 2014, pp. 109-112.

[11]. F. Fink, K. Worle, P. Gruber, A.M. Tome, J.M. Gorriz-Saez, C.G. Puntonet, and E.W. Lang, "ICA analysis of retina images for glaucoma classification", IEEE 30th Annual International Conference of the Engineering in Medicine and Biology Society, pp. 4664-4667.

[12]. K. Choudhary, and S. Wadhwa, "Glaucoma detection using cross validation algorithm", IEEE Fourth International Conference on Advanced Computing \& Communication Technologies, 2014, pp. 478-482.

[13]. R.G. Stockwell, "A basis for efficient representation of the S-transform", Digital Signal Processing, Vol. 17, No. 1, 2007, pp. 371-393. 